\title{
Shape of the Voltage-Frequency Curve Depending on the Type of the Object Detached from the QCM Surface
}

\author{
F.N. Dultsev
}

\begin{abstract}
Analysis of the shapes of voltage-frequency curves depending on the type of object detached from the surface of the quartz crystal microbalance (QCM) is carried out. It is demonstrated that the shape of the curve depends not only on the size and shape of bio-object but also on the properties of the particle. For example, a detachment of hepatitis B virus is accompanied by the fragmentation of the bio-object, and signal shape is typical for this case. In addition to a voltage value which determines the bonding force, the signal shape is also characteristic for identification of bio-object.
\end{abstract}

Keywords QCM Sensor · Rupture event scanning • Bonding forces

\section{Introduction}

The method used in this work to measure bonding forces is based on the use of the quartz crystal resonator as a sensor. Quartz crystal resonators are usually used to measure mass, that is, as quartz crystal microbalance (QCM). The addition of some mass (m) on QCM surface (AT-cut quartz plate about $100 \mu \mathrm{m}$ thick) causes a definite frequency shift $\Delta \mathrm{f}$, which can be detected by the measuring equipment [1-3]. It turned out to be possible to broaden the measuring possibilities and to enhance the sensitivity of QCM-based sensors by applying the principle of the measurement of the acoustic signal generated during the rupture of bonds holding an object on the QCM surface, as proposed in [4]. The applications of this method to the determination of phages and bacteria were demonstrated in $[5,6]$.

In those works, QCM is not only a sensor but it also plays an active role with respect to a particle bound to its surface: the dynamic increase in the voltage at the AT-cut QCM electrodes (surface) causes an increase in the amplitude of shear

\footnotetext{
F.N. Dultsev ( $\bowtie)$

Institute of Semiconductor Physics, Siberian Branch of the Russian Academy of Sciences, Novosibirsk State University, Novosibirsk, Russia

e-mail: fdultsev@isp.nsc.ru 
oscillations. Under smoothly increasing amplitude of oscillations (rupture event scanning), a particle bound to QCM surface will get detached due to inertial forces in a threshold manner, so that the force of bond rupture may be easily obtained from the threshold voltage. This procedure allows us to increase the sensitivity and to carry out reliable measurements of rupture forces of the order of $10 \mathrm{pN}$. Similarly to atomic force microscopy, rupture event scanning is a direct measurement method, it does not involve electromagnetic radiation, but rupture event scanning has much simpler instrumentation. The presence of an object on QCM surface causes a slight distortion of the shape of membrane oscillations. For the object mass $10^{-12}$ of the mass of the quartz plate, the frequency change is much smaller than that caused by the background temperature non-uniformities of quartz material. In spite of the fact that the presence or the absence of a small object on the surface cannot be recorded as mass change, the rupture moment is determined correctly because a transient process in the form of acoustic signal arises in the resonator plate. This signal points not only to the presence of analytes but also on their number, and their affinity to the receptor. In the present work, we will consider the determination of binding force on the basis of the analysis of the voltage-frequency dependence.

\section{Experimental}

AT-cut quartz plate $8.25 \mathrm{~mm}$ in diameter, plano-convex (curvature 0.2 dioptres) with the resonance frequency of $14.3 \mathrm{MHz}$ (Morion, St. Petersburg, Russia) was used in the work.

The setup is shown schematically in Fig. 1. This is the simplest arrangement. Voltage is supplied from a signal generator (1) to the QCM (2). Frequency scanning around the resonance frequency is carried out. The voltage supplied to the QCM is increased after each scanning.

Fig. 1 Electrical circuit of the experimental setup. 1 - signal generator GSS-40, 2-QCM, 3-current transformer, 4-amplifier units, 5-analog-to-digital converter, 6 - personal computer

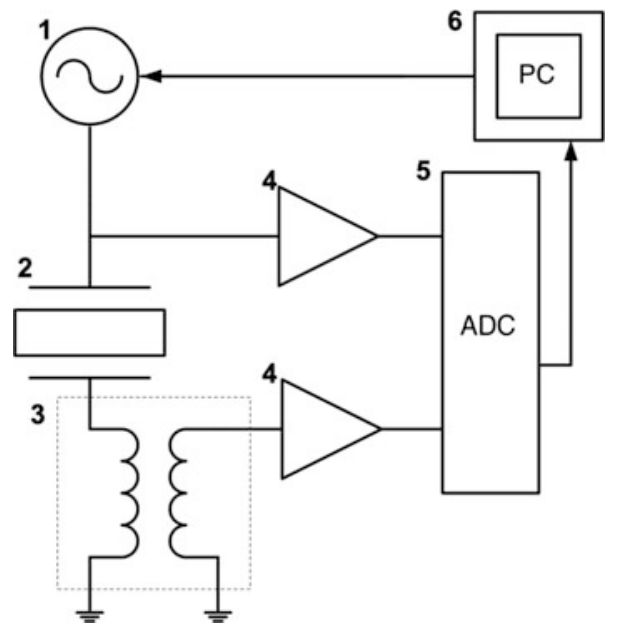


(a)

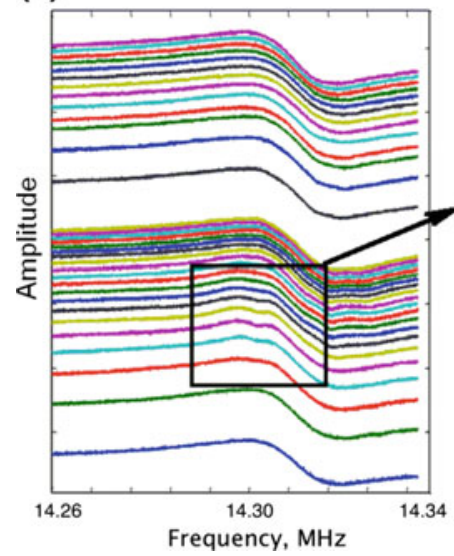

(b)

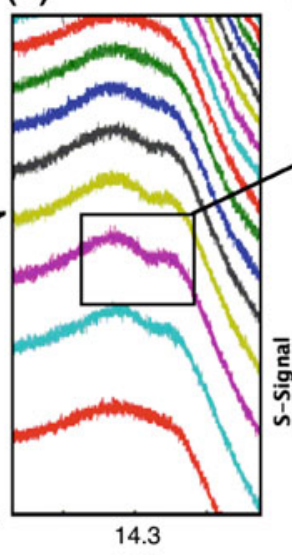

(c)

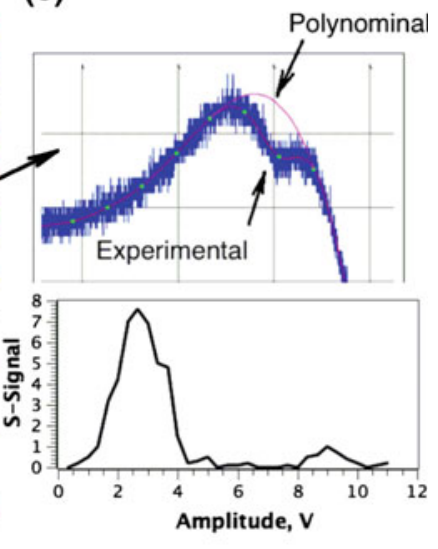

Fig. 2 The scheme explains the origin of S-signal

Voltage and the current passing through the QCM are supplied through the logarithmic amplifier (4) to the ADC (5). The software was made in MathLab. The rupture signal is recorded from the voltage-frequency dependence (VFD-method), as described in detail in [7]. Here we give a brief description of the essence of this method and illustrate it in Fig. 2a.

(a) description of the amplitude-voltage dependence for different voltage values applied to the QCM The voltage is increased step by step from bottom to top, from 0 to $10 \mathrm{~V}$. Frequency change around the resonance frequency of the QCM is plotted along the $\mathrm{X}$-axis. Distortions accompanying particle detachment (rupture) are exhibited at a definite voltage value (this region is marked in Fig. 2a and shown in more detail in Fig. 2b). S-signal is a sum of the absolute values of the integrals or the area between the experimental curve and the envelope polynomial (see upper Fig. 2c). A set of values is obtained. Figure 2c, lower part: dependence of S-signal on the amplitude of the voltage applied to the QCM. One can see that rupture occurs at the voltage of $2-4 \mathrm{~V}$; the exact position of the maximum is determined by equating to zero the derivative of the analytical parabola $y=a x^{2}+b x+c$, which is traced through the points near the maximum. The data for the unwinding of the double-stranded oligonucleotides are shown (the surface concentration is $1 \times 10^{11}$ molecules $/ \mathrm{mm}^{2}[8]$.

\section{Results and Discussion}

Mathematical processing of the experimental curves depends on the shape of these curves, which in turn depends on the object bound to the QCM surface. Some typical examples for nano-objects on QCM surface are to be considered. In this 
work, we will not deal with surface modification because the modification is carried out in a special manner for each specific case. However, in any case, the surface is prepared so that the object of interest is bound to it selectively. Specifically, bound nano-object is held by a stronger bond, and rupture occurs during scanning at a higher voltage. As an example, Fig. 3 shows the separation of a mixture of two bacterial species, one of them bound to the surface specifically and the other non-specifically.

Below we will consider signal shapes depending on the shape, mass, and a number of nano-objects on QCM surface. The simplest shape of a nano-object is a sphere. At the moment of detachment, distortions appear near the resonance at the voltage-frequency dependence. As a rule, the signal shape looks like that shown in Fig. 4.

(a)

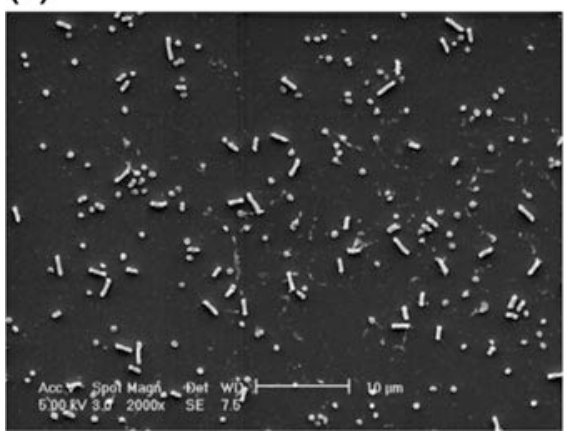

(b)

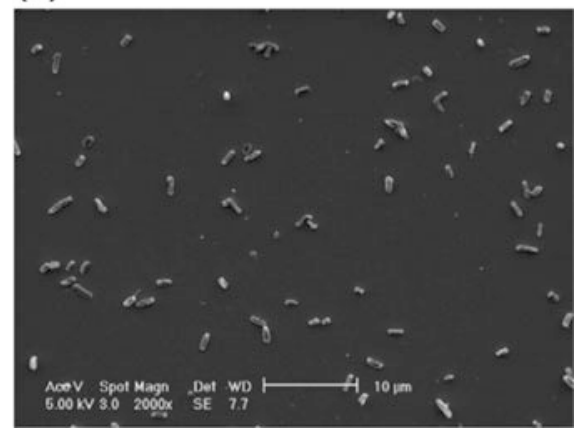

Fig. 3 (a) $20 \mu \mathrm{L}$ of an equal mixture of Staphylococcus aureus (SA) and Escherichia coli (EC) at $5 \times 10^{5}$ bacteria $\mathrm{mL}^{-1}$ incubated for $1 \mathrm{~h}$ on the QCM then washed with cold PBS. (b) QCMs treated as in (a) then scanned from 0 to $4 \mathrm{~V}$. No visible features could be discerned on a QCM coated with mAbs alone or a QCM left uncoated with protein [9]

Fig. 4 The shape of the signal accompanying the rupture of a spherical solid particle. Rupture signal is the area between the experimental and polynomial curves

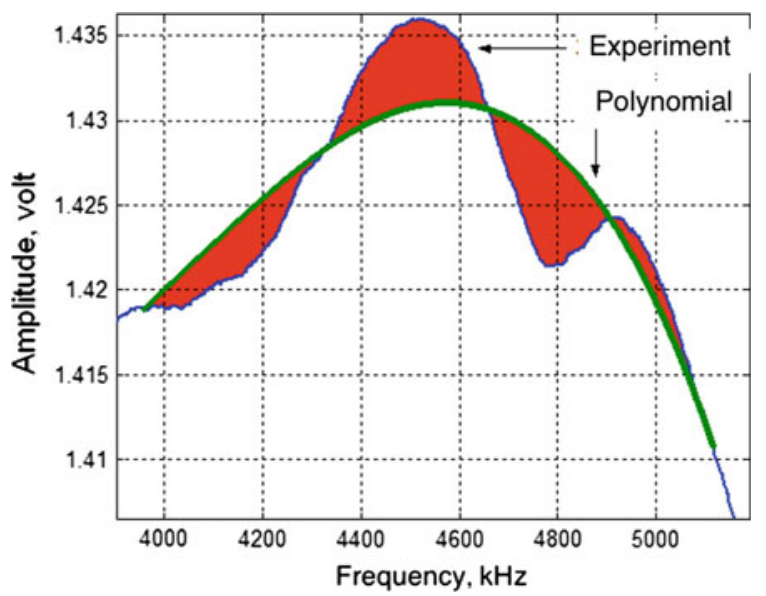


If particle mass is large, distortions are larger than those for a small particle. Testosterone linked with horseradish peroxidase was studied as an example. Testosterone provides specific bonding with QCM surface, while horseradish peroxidase has large size and mass. At the voltage about $2 \mathrm{~V}$, the molecules get detached, and strong distortion is observed in the voltage-frequency dependencies (see Fig. 5). The shape of the voltage-frequency curve does not change during further scanning.

As a rule, particles are detached within the rather narrow voltage range. For the spherical particles of the same size, for example, aerosol particles for which the particle size distribution is shown in Fig. 6, distortions are observed at one voltage value, but signal shape deviates in the form of a smooth transition, see Fig. 7. This shape of the curve is typical for the high concentration of the spherical particles with narrow size distribution.

Fig. 5 The voltagefrequency curves for the detachment of molecules with large mass

Fig. 6 Size distribution of aerosol particles obtained with the help of aerosol generator from the vapor of salicylic aldehyde
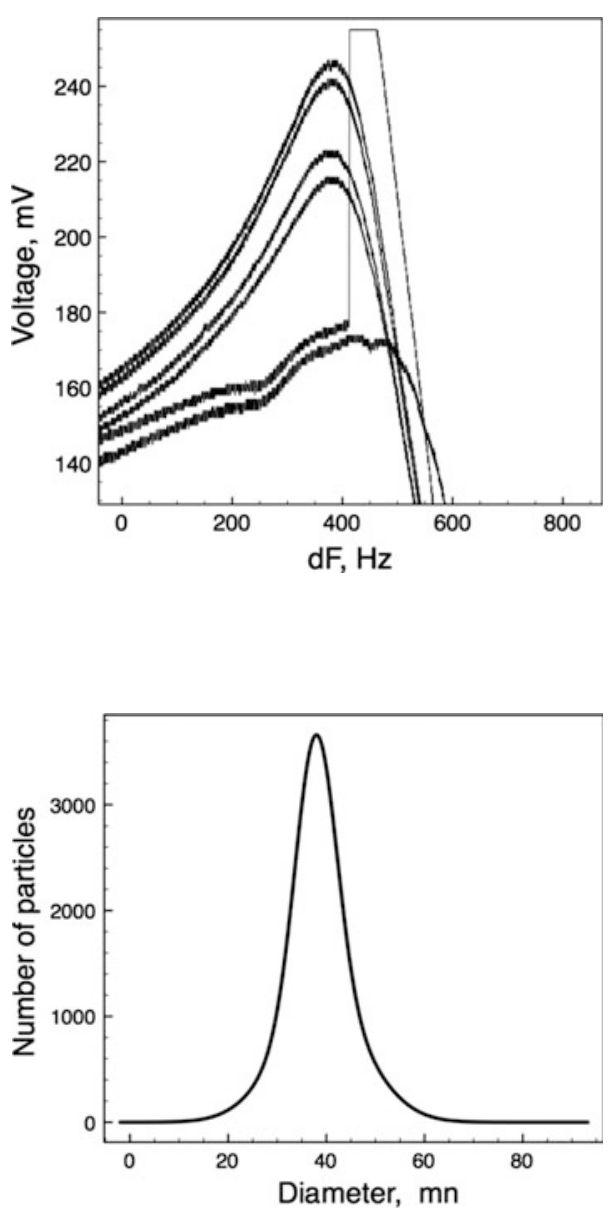

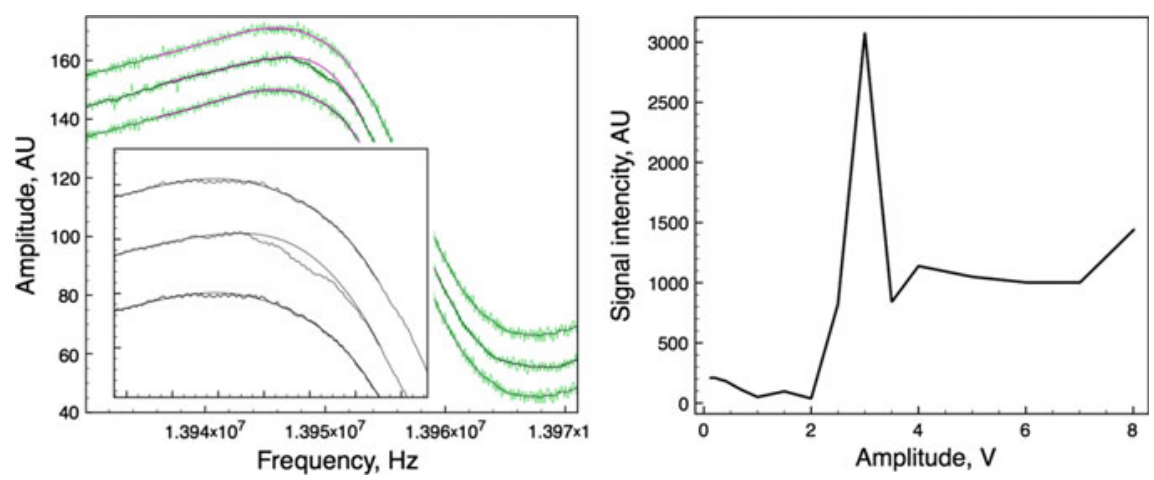

Fig. 7 Voltage-frequency dependencies (left) and the dependence of rupture signal on voltage (right) from which the bond strength may be determined. Here QCM surface with the attached amine groups $\mathrm{NH}_{2}$ is used, specific bonding is observed, which suggests that there are carboxylic groups $\mathrm{COOH}$ on the surface of aerosol particles

Rupture signal for hepatitis B virus (HBsAg) is shown in Fig. 8. A specific feature of this signal in comparison, for example, with the rupture signal of herpes virus [5] is that HBsAg rupture signal has not a narrow peak but a broad peak. This shape suggests that rupture occurs within a broad voltage range. During repeated scanning, we observe the signal at a higher voltage, and the peak becomes narrower. We used different antibodies, but the signal behavior was the same. This observation may be explained by the structure of HBsAg. It was demonstrated in the studies of HBsAg particles by means of EPR examination of spin-labeled fatty acids [10] that fatty acids in the lipid bilayer are immobilized as a consequence of the binding role of polypeptides. On the basis of the data obtained, the mechanism of HBsAg particle formation through gemmation from the membrane of endoplasmic reticulum of the host cell was proposed.

Our studies also confirm that hepatitis virus is not a rigid nanoparticle; from the viewpoint of solid state physics, it is composed of nanoparticles weakly bound with

Fig. 8 Typical curve (rupture signal) for hepatitis B virus

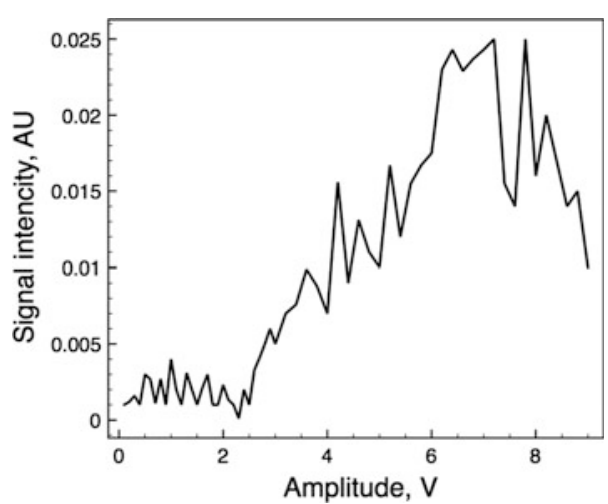


each other. So, it may be assumed that scanning causes the destruction of the virus, which we observe as a signal extended over the voltage axis.

The data for the unwinding of the double-stranded oligonucleotides (20 base pairs) are shown in Fig. 2. One can see that unwinding occurs not strictly at a definite voltage but within some interval (see Fig. 2c) - from 2 to $4 \mathrm{~V}$.

\section{Conclusions}

It is demonstrated in the work how the properties of the particle detached from the QCM surface are connected with the shape of the rupture signal. A sharp single peak is observed as a result of the rupture of a rigid spherical particle. Distortions are observed on one voltage-frequency dependence, while peak broadening is observed in the case of the separation of long nano-objects (dsDNA) linked along their length. If a nanoparticle composed of separate smaller particles is detached from the surface, fragmentation of this particle occurs, and rupture signal as a set of separate peaks is observed.

QCM appears as an element functioning as an active resonator, absorbing surface, and signal recording unit. This allows us to use QCM as a tool to detect particles bound to the surface with different forces, in particular in medical diagnostics and in sensing. The time of sensor recovery is exactly measuring time because measurement involves purification of the sensor surface. An increase in sensitivity does not cause a decrease in the selectivity of the instrument.

Acknowledgements Research is carried out with the financial support of the state represented by the Ministry of Education and Science of the Russian Federation. Agreement No. 14.607.21.0125 from 27.10.2015. Unique project Identifier: RFMEFI60715X0125.

\section{References}

1. Sauerbray, G.Z.: Use of quartz vibrator for weighing thin films on a microbalance. Z. Phys. 155, 206-212 (1959)

2. Mecea, V.M.: Quartz crystal microbalance to fundamental principles of mass measurements. Anal. Lett. 38, 753-767 (2005)

3. Marx, K.A.: Quartz crystal microbalance: a useful tool for studying thin polymer films and complex biomolecular systems at the solution-surface interface. Biomacromolecules 4(5), 1099-1119 (2003)

4. Dultsev, F.N., Ostanin, V.P., Klenerman, D.: "Hearing the bond breakage". Measurement of bond rupture forces using a quartz crystal microbalance. Langmuir 16, 5036-5040 (2000)

5. Cooper, M.A., Dultsev F.N., Ostanin V.P., et al. Direct and sensitive detection of a human virus by rupture event scanning// Nat. Biotechnol. 2001. N 19. p. $833-837$

6. Dultsev, F.N., Speight, R.E., Fiorini, M.T., et al.: Direct and quantitative detection of bacteriophage by "hearing" surface detachment using a quartz crystal microbalance. Anal. Chem. 73, 3935-3939 (2001) 
7. Dultsev, F.N., Kolosovsky, E.A., Mik, I.A.: New procedure to record the rupture of bonds between macromolecules and the surface of the quartz crystal microbalance (QCM). Langmuir 28, 13793-137978 (2012)

8. Dultsev, F.N., Kolosovsky, E.A., Mik, I.A., et al.: QCM-based measurement of bond rupture forces in DNA double helices for complementarity sensing. Langmuir 30(13), 3795-3801 (2014)

9. Cooper, M.A., Dultsev, F.N., Ostanin, V.P., Klenerman, D.: Separation and detection of bacteria using rupture event scanning. Analytica Chimica Acta. 702, 233- 238 (2011)

10. Satoh, O., Imai, H., Yoneyama, T., et al.: Membrane structure of the hepatitis B virus surface antigen particle. J. Biochem. 127, 543-550 (2000)

Open Access This chapter is licensed under the terms of the Creative Commons Attribution 4.0 International License (http://creativecommons.org/licenses/by/4.0/), which permits use, sharing, adaptation, distribution and reproduction in any medium or format, as long as you give appropriate credit to the original author(s) and the source, provide a link to the Creative Commons license and indicate if changes were made.

The images or other third party material in this chapter are included in the chapter's Creative Commons license, unless indicated otherwise in a credit line to the material. If material is not included in the chapter's Creative Commons license and your intended use is not permitted by statutory regulation or exceeds the permitted use, you will need to obtain permission directly from the copyright holder.

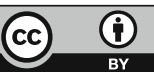

